\title{
Variabilité infraspécifique du retrait après reconditionnement, du rendement papetier et de la longueur des fibres, chez Eucalyptus camaldulensis dans deux dispositifs italiens
}

\author{
A Sesbou $^{1 *}$, G Nepveu ${ }^{2}$ \\ ${ }^{1}$ École Nationale Forestière d'Ingénieurs, Salé, Maroc ; \\ ${ }^{2}$ INRA, centre de recherches de Nancy, station de recherches sur la qualité des bois, \\ Champenoux, 54280 Seichamps France.
}

(reçu le 7 mars 1988 ; accepté le 11 octobre 1989)

Résumé - Vingt-quatre provenances australiennes d'Eucalyptus camaldulensis installées dans 2 dispositifs italiens (Sicile et Calabre) ont été étudiées du point de vue des retraits après reconditionnement, du rendement papetier et de la longueur des fibres en relation avec la vigueur. Ces caractères ont été mesurés sur carottes de sondage ( 1200 échantillons). Les principaux résultats sont les suivants :

- La variabilité infraspécifique est très importante pour les caractères étudiés. Le retrait volumétrique après reconditionnement varie selon les provenances et la station de 10 à $19 \%$, le rendement papetier de 39,8 à $43,9 \%$, la longueur des fibres de 0,69 à $0,82 \mathrm{~mm}$.

- Le reconditionnement a un effet bénéfique sur la récupération du collapse. II fait passer le retrait volumétrique de 60 à $18 \%$ pour la plus mauvaise provenance et de 22 à $12 \%$ pour la meilleure.

- Une grande région de provenances homogène, le bassin de la rivière Murray, a été mise en évidence ; elle se caractérise par des retraits après reconditionnement et une vigueur élevés et une longueur des fibres faible.

- La variabilité de la production papetière est spécialement étudiée en envisageant la production par unité de poids de bois anhydre, ou de volume de bois vert ou encore par arbre. - L'effet station est très significatif pour la vigueur et le rendement papetier. Quand on passe de Sicile en Calabre, la vigueur augmente, le rendement papetier diminue et le retrait volumétrique après reconditionnement reste comparable.

- Les interactions génotype $x$ environnement sont faibles; elles sont dues à l'instabilité de quelques provenances seulement.

Eucalyptus camaldulensis / varlabilité infraspécifique / Interaction génotype $\times$ envlronnement / rendement papetier / longueur des fibres / retrait après reconditionnement

\footnotetext{
* Correspondance et tirés à part
} 


\begin{abstract}
Summary - Infraspeciflc variabllity of collapse-free shrinkage, pulp yleld and flbre length for Eucalyptus camaldulensis evaluated in two Itallan experiments. Twenty-four Eucalyptus camaldulensis provenances from 2 experimental designs located in Italy (Sicily and Calabria) were investigated for collapse-free shrinkage, pulp yield and fibre length with regard to the vigour. The characteristics were measured on a sampling of 1200 increment cores.

The main results are as follows:

- The infraspecific variability (table II) is large. The means of different characteristics studied are reported in tables $I I I$ and $I V$. Depending on the provenance and geographical location, the volumetric shrinkage can vary from $10-19 \%$, the pulp yield from $39.8-43.9 \%$ and the fibre length from $0.69-0.82 \mathrm{~mm}$.

- The reconditioning has a very positive effect on the collapse recuperation. Volumetric shrinkage decreases from $60 \%$ before reconditioning to $18 \%$ after this treatment for the bad provenance and from $22-12 \%$ respectively for the best one.

- A large homogeneous region of provenances, the Murray River System, was identified. It is characterized (table $V$ ) by high shrinkage without collapse, high vigour and low fibre length.

- The variability of the pulp fibre production is specially studied as a function of the provenances used in regard to the production per oven-dry weight, green wood volume and tree (figs 1 and 2).

- The location effect (table VII) is important for vigour and pulp yield only. These characteristics are higher in Calabria.

- The interaction between genotype and environment (table VIII) appears to be weak; it is mainly due to the instability of a few provenances only.
\end{abstract}

Eucalyptus camaldulensis / infraspecific variability / genotype $\times$ environment interaction / pulp yield / fibre length / collapse-free shrinkage

\section{INTRODUCTION}

Lacaze (1978) a souligné que «l'étude de la variabilité infraspécifique est un préalable souvent obligatoire à tout programme de sélection individuelle. On peut, en effet, douter de l'efficacité d'un programme de creation de variété synthétique constituée à partir de géniteurs choisis dans une population (espèce, provenance) elle-même génétiquement inférieure». L'intérêt de l'étude de la variabilité infraspécifique est donc évident pour la conduite d'un bon programme d'amélioration. Destremau et al (1973), Bellefontaine et al (1977), Lacaze (1977) ont montré que cette étude est concluante pour les caractères de vigueur dans le cas de l'Eucalyptus camaldulensis.

On constate que cette essence n'a pas l'attention qu'elle mérite de la part des utilisateurs et jusqu'à présent, peu d'études systématiques ont été faites sur la qualité du bois. Ceci est particulièrement dommageable dans la mesure où de nombreux pays qui avaient pris une optique papetiere pour leurs reboisements d'Eucalyptus envisagent de plus en plus une utilisation en bois d'œuvre, donc doivent se pencher sur certains critères très importants dans cette perspective, tels la rétractibilité du bois. La variabilité génétique infraspécifique de la qualité du bois de cette espèce ainsi que l'héritabilité n'ont pratiquement jamais été étudiees à l'exception des travaux de Rudman (1970) sur un échantillonnage réduit alors que l'amélioration génétique pour la croissance est en progrès constant. Or, de nombreux auteurs (Polge et Illy, 1967 ; Polge 1973 ; Janin et Rahme, 1972 ; Keller, 1973; Keller et Thoby, 
1977; Nanson et al, 1975a, 1975b ; Nepveu, 1973, 1986) ont montré l'importance du contrôle génétique sur certaines caractéristiques technologiques en plus de la vigueur, de l'adaptabilité et l'intérêt qu'il pouvait y avoir à sélectionner pour les critères de qualité du bois ou au moins à en connaître la variation.

Dans un précédent article (Sesbou et Nepveu, 1978), nous avons étudié la variabilité infraspécifique du retrait sans reconditionnement et de la densité du bois chez cette espèce. Elle s'était d'ailleurs révélee considerable.

Dans la présente étude, nous allons compléter ce travail en appréciant la variabilité infraspécifique ainsi que l'interaction génotype $x$ environnement pour d'autres caractères dont l'amélioration est primordiale pour une meilleure utilisation de cette essence : retrait après reconditionnement, rendement papetier et longueur des fibres.

Les caractéristiques papetières, le rendement en pâtes et la longueur des fibres, sont parmi les critères les plus importants pour la définition de l'aptitude papetière d'une essence. En effet, pour des raisons de rentabilité économique, la capacité des usines papetières devient de plus en plus grande alors que les superficies forestières ne peuvent plus guère être augmentées. II convient donc d'augmenter la quantité de matière sèche et son rendement à l'hectare dans les zones plantées.

\section{MATÉRIEL ET MÉTHODE}

\section{Matériel}

Vingt-quatre provenances d'Eucalyptus camaldulensis prélevees dans 2 stations italiennes respectivement en Sicile et en Calabre ont été utilisées. Au moment des sondages, les arbres étaient âgés de 9 ans. Le tableau I donne la liste des provenances utilisées ainsi que leurs régions d'origine dans l'aire naturelle australienne. Le dispositif expérimental est le meme pour les 2 stations. Il est a base de blocs incomplets équilibrés. Ses caractéristiques sont les suivantes:

- Nombre de provenances : 25 (la provenance 7116 a été séparée en 2 lots)

- Nombre de répétitions : 6

- Nombre de blocs par répétition : 5

- Nombre de parcelles unitaires par bloc : 5

- Nombre d'arbres par parcelle unitaire : 25

Dans chaque parcelle unitaire, on a choisi au hasard 2 arbres sur lesquels on a prélevé 2 demi-carottes ( 1 de chaque côté de l'arbre) espacées de 20 à $30 \mathrm{~cm}$.

\section{Méthode :}

Sur les carottes de sondage, les caractères suivants ont été mesurés ou calculés :

Les retraits longitudinal, tangentiel et radial du bois après reconditionnement entre les états saturé et sec à l'air, les 2 premiers étant obtenus à l'aide d'un capteur spécialement adapté à la mesure des carottes de sondage (Sesbou, 1977 ; Ferrand, 1981). Le reconditionnement du bois pour la récupération du collapse a consisté en un traitement des carottes, à l'état sec à l'air, à la vapeur a $120^{\circ} \mathrm{C}$ pendant $15 \mathrm{~min}$ environ.

- Le retrait volumétrique après reconditionnement calculé à partir des 3 retraits précédents (Sesbou, 1977, op cit).

- Le rendement papetier obtenu par la méthode des microcuissons (Janin, 1972) avec les parametres suivants : $1 \mathrm{~h} 30$ pour la montée de température de $20-170^{\circ} \mathrm{C}$; palier de $1 \mathrm{~h} 30$ à $170{ }^{\circ} \mathrm{C}$; taux d'alcali actif : $22 \%$; taux de sulfidite : $25 \%$ et rapport liqueur/bois : 4 .

- La longueur moyenne des fibres obtenue en faisant la moyenne des mesures de 50 fibres colorées a la safranine et agrandies 240 fois. On s'est limité à 7 échantillons par provenance et par station.

II faut signaler que nous disposions également des circonferences sur écorce à $1,30 \mathrm{~m}$ et des hauteurs des arbres à l'âge de 9 ans. 
Tableau I. Description des provenances.

\begin{tabular}{|c|c|c|c|c|c|c|}
\hline No & Noms des provenances & Sous-région & Région & $\begin{array}{l}\text { Alti- } \\
\text { tude } \\
(m)\end{array}$ & $\begin{array}{l}\text { uviométrie } \\
(\mathrm{mm})\end{array}$ & $\begin{array}{l}\text { Saison } \\
\text { seche }\end{array}$ \\
\hline $1=6788 \mid$ & Todd river, Alice Springs & sud & $\begin{array}{l}\text { Territoire } \\
\text { du Nord }\end{array}$ & $\mid \begin{array}{ll}\mid 579 & 1 \\
1 & \mid\end{array}$ & 246 & Hiver \\
\hline $2=6843 \mid$ & Shepparton & Rivière & Bassin de la & $|152|$ & 418 & Etè \\
\hline $3=6844$ & woohlpooer state forest & Murray & la rivière & $|300|$ & 597 & Etè \\
\hline ........ & $\ldots \ldots \ldots \ldots \ldots \ldots \ldots, n$ & $\ldots \ldots \ldots \ldots \ldots$ & Murray & & & \\
\hline $4=6845$ & Lake Albacutya & Lac salé | & & $|183|$ & 341 & Eté \\
\hline $5=6869 \mid$ & Katherine & Nord & $\begin{array}{l}\text { Territoire } \\
\text { du Nord }\end{array}$ & $\mid \begin{array}{ll}1 & 110 \\
\mid & \mid\end{array}$ & $943 \quad 1$ & Hiver \\
\hline$==x== \pm=\mid=$ & 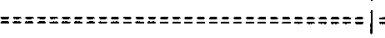 & 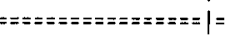 & 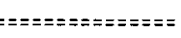 & $=1====\mid=$ & $z==== \pm==-1=$ & 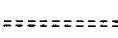 \\
\hline $6=6870$ & Eulo & \begin{tabular}{l|} 
Riviere \\
Paroo
\end{tabular} & $\begin{array}{l}\text { Australie } \\
\text { meridionale }\end{array}$ & $|183|$ & 192 & Eté \\
\hline$=====-=1=$ & 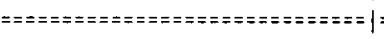 & 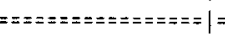 & 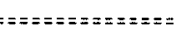 & $=1====1=$ & $====-=\pi=\mid=$ & $=======-$ \\
\hline $7=6948$ & Bullock Creek & Bassin Lac Eyre I & Queens I and & $|457|$ & $456 \quad \mid$ & Hiver \\
\hline $8=6953$ & Petford & 1 & Nord & $|510|$ & 704 & Hiver \\
\hline & & Rivière Darting & & $|\cdots \cdots|$ & & \\
\hline $\begin{array}{l}9=6958 \mid \\
\ldots . . . . . .\end{array}$ & $\begin{array}{l}\text { Bourke } \\
\ldots \ldots \ldots \ldots \ldots \ldots\end{array}$ & Riviere Darling | & & $\left|\begin{array}{c}|110| \\
\mid \cdots \cdots\end{array}\right|$ & 904 & $\begin{array}{l}\text { Etc } \\
\cdots\end{array}$ \\
\hline $10=6966 \mid$ & Est d'Adélaide, Menger's Hilll & Hill site & Australie & $600 \mid$ & 1163 & Eté \\
\hline $11=6966$ & " & i & méridionale & $1 " 1$ & $"$ & $"$ \\
\hline $12=6966$ & $"$ & 1 & & $" 1$ & $" 1$ & $"$ \\
\hline $13=6966$ & $"$ & i & & $" 1$ & $"$ & $"$ \\
\hline $14=6966$ & $"$ & | & & $" 1$ & $"$ & $"$ \\
\hline $15=6966$ & $"$ & 1 & & $" 1$ & $"$ & $"$ \\
\hline $16=6966 \mid$ & " & | & et & $1 " 1$ & $"$ & $"$ \\
\hline & 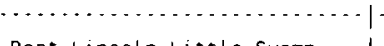 & 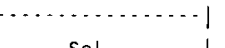 & & 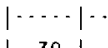 & $-1 \cdot$ & $\cdots$ \\
\hline $\begin{array}{l}17=6975 \mid \\
18=6978\end{array}$ & Port Lincoln, Little Swamp & Sol & & 301 & 456 & Ete \\
\hline $\begin{array}{l}18=6978 \mid \\
\cdots-\cdots\end{array}$ & $\begin{array}{c}1 " \\
\ldots\end{array}$ & calcaire & $\begin{array}{l}\text { Bassin de } \\
\text { la Rivière }\end{array}$ & $\mid \begin{array}{ll}1 & \mid \\
\mid \cdots \cdots & \mid-1\end{array}$ & $\ldots \ldots+1$ & $\cdots \cdot$ \\
\hline $\begin{array}{l}19=6990 \mid \\
\ldots \ldots \ldots \mid-\end{array}$ & Darlington Point (NSW) & R.Murrumbidgee & Murray & $\left|\begin{array}{c|}91 \mid \\
\mid \cdots \cdots\end{array}\right|$ & 379 & Ete \\
\hline $20=6991 \mid$ & Walpola Island St.Forest ( $V$ ) I & Rivière Murray | & & $1 \quad 1$ & 243 & Eté \\
\hline $21=7029$ & Murchison River & Northern Gold & Australie & $|213|$ & 465 & Ete \\
\hline $22=7046 \mid$ & Wiluna - Gum Creek & Fields & Occidentale & $|480|$ & 245 & Ete \\
\hline $23=7080$ & New castle waters Creek & Nord & Territoire & $|213|$ & 475 & Hiver \\
\hline $26=7116$ & Tennant Creek, Goodairs Creck I & sud & $d u$ & $|330|$ & 340 & Hiver \\
\hline $25=7116$ & $\begin{array}{llll}\ldots & \ldots & 1\end{array}$ & sud & Nord & | $" 1$ & $"$ & $"$ \\
\hline
\end{tabular}




\section{RÉSULTATS ET DISCUSSION}

\section{Variabllité infraspécifique}

\section{Analyse de la variance pour les dif- férents caractères étudiés.}

Nous allons étudier, dans chaque station, les effets "provenance" (24 degrés de liberté), "répétition" (5 degrés de liberté) et l'interaction "provenance $x$ répétition" (120 degrés de liberté). Le tableau II donne les résultats de l'analyse de variance et les tableaux III et IV donnent, pour les caractères étudiés pour les essais de Sicile et de Calabre, les moyennes des provenances, la moyenne générale ainsi que le coefficient de variation interprovenance.
Nous avons fait figurer dans ces 2 derniers tableaux les données concernant le retrait volumétrique et l'infradensité du bois (Sesbou et Nepveu, 1978 op cit) auxquelles nous ferons allusion lors de la discussion des résultats.

\section{Effet provenance :}

le tableau II montre que l'effet provenance est très significatif pour tous les caractères étudiés, à l'exception du retrait longitudinal après reconditionnement et de la circonférence en Calabre. On remarque une variabilité importante pour le retrait volumétrique après reconditionnement qui varie de 10,0 à $19,1 \%$ en Sicile et de 9,5 à $18,8 \%$ en Calabre. Les coefficients de variation interprovenance pour ce caractere

Tableau II. Résultats des analyses de variance pour les effets provenance, répétition, interaction provenance $x$ répétition; Valeurs des $\mathrm{F}$ calculés et signification.

" : effet significatif au seuil de 5\%; ${ }^{\star *}$ : effet significatif au seuil de $1 \%$; NS : effet non significatif au seuil de $5 \%$.

\begin{tabular}{|c|c|c|c|c|}
\hline \multirow[b]{2}{*}{ Caracteres } & \multicolumn{4}{|c|}{ Sources de variation } \\
\hline & Station & Provenance & Répetition & Interaction \\
\hline $\begin{array}{l}\text { Retrait longitudinal } \\
\text { apres reconditionnement (RLC) }\end{array}$ & $\begin{array}{l}\text { Sicile } \\
\text { Calabre }\end{array}$ & $\begin{array}{l}3,35 * * \\
1,14 \text { NS }\end{array}$ & $\begin{array}{l}7,44^{\star \star} \\
3,14 \text { ** }\end{array}$ & $\begin{array}{l}1,27 * \\
0,85 \mathrm{NS}\end{array}$ \\
\hline $\begin{array}{l}\text { Retrait tangentiel } \\
\text { apres reconditionnement (RTC) }\end{array}$ & $\begin{array}{l}\text { Sicile } \\
\text { Calabre }\end{array}$ & $\begin{array}{l}25,34^{\star \star} \\
12,33^{\star \star}\end{array}$ & $\begin{array}{l}2,57 * * \\
3,48 * *\end{array}$ & $\begin{array}{l}1,28 * \\
1,71^{* *}\end{array}$ \\
\hline $\begin{array}{l}\text { Retrait radial } \\
\text { après reconditionnement (RRC) }\end{array}$ & $\begin{array}{l}\text { Sicile } \\
\text { Calabre }\end{array}$ & $\begin{array}{l}4,68 * * \\
6,10^{* *}\end{array}$ & $\begin{array}{l}3,10 * \star \\
0,75 \mathrm{NS}\end{array}$ & $\begin{array}{l}1,53 * * \\
1,10 \mathrm{NS}\end{array}$ \\
\hline $\begin{array}{l}\text { Retrait volumétrique } \\
\text { après reconditionnement (RVC) }\end{array}$ & $\begin{array}{l}\text { Sicile } \\
\text { Calabre }\end{array}$ & $\begin{array}{l}19,89 * * \\
11,44 * *\end{array}$ & $\begin{array}{l}3,65 * * \\
2,69 *\end{array}$ & $\begin{array}{l}1,29^{*} \\
1,63^{\star *}\end{array}$ \\
\hline Rendement papetier (Rdt) & $\begin{array}{l}\text { Sicile } \\
\text { Calabre }\end{array}$ & $\begin{array}{l}6,82 * * \\
7,79 * *\end{array}$ & $\begin{aligned} 1,19 \mathrm{NS} \\
11,62 \text { ** }\end{aligned}$ & $\begin{array}{l}1,74^{* *} . \\
2,92^{* *}\end{array}$ \\
\hline Longueur des fibres (LF) & $\begin{array}{l}\text { Sicile } \\
\text { Calabre }\end{array}$ & $\begin{array}{l}2,78^{* *} \\
1,87^{*}\end{array}$ & $\begin{array}{l}1,09 \mathrm{NS} \\
t, 46 \mathrm{NS}\end{array}$ & $\begin{array}{l}0,98 \text { NS } \\
1,72 *\end{array}$ \\
\hline Circonférence $\left(C_{1,30}\right)$ & $\begin{array}{l}\text { Sicile } \\
\text { Calabre }\end{array}$ & $\begin{array}{l}4,59 * * \\
1,57 \text { NS }\end{array}$ & $\begin{array}{l}1,69 \text { NS } \\
2,00 \mathrm{NS}\end{array}$ & $\begin{array}{l}0,66 \mathrm{NS} \\
0,92 \mathrm{NS}\end{array}$ \\
\hline Hauteur $(\mathrm{H})$ & $\begin{array}{l}\text { Sicile } \\
\text { Calabre }\end{array}$ & $\begin{array}{l}9,60 * \star \\
2,22 * \star\end{array}$ & $\begin{array}{l}5,04 * \star \\
3,04 *\end{array}$ & $\begin{array}{l}1,43^{*} \\
0,96 \text { NS }\end{array}$ \\
\hline
\end{tabular}


Tableau III. Moyennes des provenances, moyennes générales et coefficients de variation pour les différents caractères en Sicile.

\begin{tabular}{|c|c|c|c|c|c|c|c|c|c|c|}
\hline Provenances & $\begin{array}{l}R V \\
(\%)\end{array}$ & $\begin{array}{l}R L C \\
(\%)\end{array}$ & $\begin{array}{l}R T C \\
(\%)\end{array}$ & $\begin{array}{c}R R C \\
(\%)\end{array}$ & $\begin{array}{l}R V C \\
(\%)\end{array}$ & $\begin{array}{c}D_{b} \\
\left(k g / m^{3}\right)\end{array}$ & $\begin{array}{l}R d t \\
(\%)\end{array}$ & $\begin{array}{c}L F \\
(\mu m)\end{array}$ & $\begin{array}{l}C_{1,30} \\
(\mathrm{~cm})\end{array}$ & $\underset{(m)}{H}$ \\
\hline $1=6788$ & 38,3 & 0,49 & 8,5 & 3,1 & 12,4 & 528 & 41,5 & 734 & 32,9 & 7,1 \\
\hline $2=6843$ & 57,80 & 0,21 & 11,8 & 2,8 & 15,1 & 454 & 43,2 & 729 & 41,1 & 9,1 \\
\hline $3=6844$ & 51,90 & 0,25 & 10,5 & 2,8 & 13,9 & 451 & 41,2 & 710 & 43,7 & 9.0 \\
\hline $4=6845$ & 49,58 & 0,25 & 11,6 & 3,1 & 15,3 & 502 & 41,8 & 745 & 40,1 & 9,9 \\
\hline $5=6869$ & 30,55 & 0,22 & 8,6 & 2,9 & 12,1 & 506 & 41,7 & 769 & 32,3 & 7,5 \\
\hline $6=6870$ & 44,59 & 0,27 & 9,7 & 2,9 & 13,2 & 500 & 41.4 & 723 & 39,0 & 8,2 \\
\hline $7=6948$ & 27,11 & 0,34 & 7.6 & 2,7 & 10,9 & 520 & 41,0 & 744 & 31,7 & 7,0 \\
\hline $8=6953$ & 24,26 & 0,23 & 8,5 & 2,9 & 12,0 & 534 & 43,7 & 770 & 33,1 & 8,3 \\
\hline $9=6958$ & 45,14 & 0,19 & 11,4 & 3,0 & 15,0 & 504 & 41,7 & 768 & 33,1 & 8,0 \\
\hline $10=6966$ & 59,93 & 0,45 & 13,8 & 3,0 & 17.9 & 482 & 40,7 & 720 & 38,9 & 8,3 \\
\hline $11=6968$ & 56,45 & 0,56 & 13,1 & 3,2 & 17.4 & 490 & 41.3 & 730 & 42,5 & 9,4 \\
\hline $12=6969$ & 54,92 & 0,44 & 14.7 & 3,3 & 19,1 & 504 & 41,3 & 748 & 39.0 & 9.2 \\
\hline $13=6970$ & 42,49 & 0,42 & 11,4 & 2,9 & 15,2 & 535 & 40,2 & 759 & 39,4 & 9,0 \\
\hline $14=6971$ & 47,47 & 0.54 & 13.2 & 3.7 & 18,1 & 505 & 41,5 & 727 & 39.6 & 9.0 \\
\hline $15=6973$ & 53,18 & 0,30 & 10,7 & 2,2 & 13,5 & 461 & 40,7 & 689 & 38,9 & 7,9 \\
\hline $16=6974$ & 55,72 & 0,32 & 12,8 & 3,3 & 17,0 & 465 & 41,2 & 740 & 41,5 & 9,2 \\
\hline $17=6975$ & 55,32 & 0,44 & 12,1 & 2.9 & 15,8 & 463 & 43.9 & 725 & 33,4 & 7,6 \\
\hline $18=6978$ & 52,36 & 0.40 & 13,0 & 3,2 & 17,2 & 468 & 42,7 & 738 & 40,2 & 7,7 \\
\hline $19=6990$ & 56.29 & 0,29 & 10,7 & 2,5 & 13,9 & 455 & 40,0 & 725 & 41,0 & 8,9 \\
\hline $20=6991$ & 43,52 & 0,20 & 9,8 & 2,8 & 13,2 & 495 & 43,0 & 739 & 35,2 & 8,6 \\
\hline $21=7029$ & 27,12 & 0.27 & 7,9 & 2,6 & 11,0 & 536 & 41,5 & 761 & 34.7 & 7.9 \\
\hline $22=7046$ & 27,23 & 0,15 & 7,5 & 2,1 & 10,0 & 506 & 41,3 & 788 & 34,1 & 7,4 \\
\hline $23=7080$ & 26,68 & 0,48 & 7,5 & 2,7 & 11,0 & 542 & 40,6 & 747 & 29,4 & 5,9 \\
\hline $24=7116 \mathrm{~A}$ & 21,90 & 0,31 & 8,1 & 3,3 & 12,0 & 584 & 40,7 & 770 & 30,5 & 6,0 \\
\hline $25=71168$ & 23,72 & 0,26 & 8,0 & 3,4 & 11,9 & 581 & 41,2 & 780 & 28,1 & 6,4 \\
\hline Moyennes & 42,9 & 0,33 & 10,5 & 2,9 & 14,2 & 503 & 41,6 & 743 & 36,5 & 9,9 \\
\hline $\mathrm{CV}_{p}(\%)$ & 30,2 & 35,4 & 21,3 & 12,1 & 18,4 & 7,3 & 2,4 & 3,1 & 12,2 & 13,4 \\
\hline
\end{tabular}

sont très élevés : $18,4 \%$ pour la Sicile et $19,0 \%$ pour la Calabre. Cette variabilité existe pour les retraits linéaires dans les 3 directions d'anisotropie du bois pour lesquels les coefficients de variation sont élevés, notamment celui du retrait longitudinal qui atteint 35,4\% en Sicile et $47,4 \%$ en Calabre.

Pour les propriétés papetières, nous avons un rendement papetier, qui varie entre 40,0 et $43,9 \%$ en Sicile, 39,8 et $42,7 \%$ en Calabre et une longueur des fibres qui donne les moyennes de provenances extrêmes suivantes : $0,689 \mathrm{~mm}$ et $0,788 \mathrm{~mm}$ pour la Sicile, $0,697 \mathrm{~mm}$ et $0,823 \mathrm{~mm}$ pour la Calabre. Les coefficients de variation interprovenance sont faibles $2,4 \%$ en Sicile et $2,0 \%$ en Calabre pour le rendement papetier, $3,1 \%$ en Sicile et $3,5 \%$ en $\mathrm{Ca}$ - 
Tableau IV. Moyennes des provenances, moyennes générales et coefficients de variation pour les différents caractères en Calabre.

\begin{tabular}{|c|c|c|c|c|c|c|c|c|c|c|}
\hline Provenances & $\begin{array}{l}R V \\
(\%)\end{array}$ & $\begin{array}{l}\text { RLC } \\
(\%)\end{array}$ & $\begin{array}{l}\text { RTC } \\
(\%)\end{array}$ & $\begin{array}{c}R R C \\
(\%)\end{array}$ & $\begin{array}{l}\text { RVC } \\
(\%)\end{array}$ & $\begin{array}{c}D_{b} \\
\left(\mathrm{~kg} / \mathrm{m}^{3}\right)\end{array}$ & $\begin{array}{l}R d t \\
(\%)\end{array}$ & $\begin{array}{c}L F \\
(\mu m)\end{array}$ & $\begin{array}{l}C_{1,30} \\
(\mathrm{~cm})\end{array}$ & $\begin{array}{c}H \\
(m)\end{array}$ \\
\hline $1=6788$ & 28,3 & 0,16 & 7,6 & 2,5 & 10,6 & 553 & 41,3 & 748 & 39.9 & 11,76 \\
\hline $2=6843$ & 50,0 & 0,25 & 11,2 & 2,2 & 13,9 & 440 & 42,7 & 747 & 41,5 & 10,87 \\
\hline $3=6844$ & 49,3 & 0,36 & 10,4 & 3,1 & 14,3 & 457 & 41,3 & 740 & 45,3 & 11,84 \\
\hline $4=6845$ & 43,2 & 0,17 & 9.5 & 2,9 & 13,1 & 513 & 40,8 & 722 & 46,1 & 13,07 \\
\hline $5=6869$ & 25,9 & 0,44 & 9,1 & 3,8 & 13,8 & 547 & 40,3 & 762 & 38,8 & 10,10 \\
\hline $6=6870$ & 35,4 & 0,18 & 8,2 & 3,0 & 11.7 & 517 & 41,0 & 728 & 36,2 & 10,18 \\
\hline $7=6948$ & 21,9 & 0,32 & 7,8 & 3,4 & 11,8 & 552 & 40,8 & 755 & 36,1 & 11,57 \\
\hline $8=6953$ & 21,4 & 0,24 & 8,2 & 3,4 & 12,2 & 551 & 42,4 & 823 & 40,8 & 12,92 \\
\hline $9=6958$ & 38,3 & 0,16 & 9,4 & 3,0 & 12,9 & 491 & 40,2 & 723 & 42,4 & 11,17 \\
\hline $10=6966$ & 45,7 & 0,38 & 11,2 & 3,5 & 15,6 & 495 & 40,2 & 723 & 42,4 & 11,17 \\
\hline $11=6968$ & 44,6 & 0,60 & 10,5 & 3,4 & 14,9 & 489 & 40,4 & 423 & 41,8 & 11,10 \\
\hline $12=6969$ & 40,1 & 0,46 & 11,1 & 3,3 & 5,3 & 529 & 40,6 & 773 & 39,9 & 11,39 \\
\hline $13=6970$ & 42,7 & 0.43 & 11.6 & 3,9 & 15.5 & 513 & 40,1 & 756 & 42,9 & 12,00 \\
\hline $14=6971$ & 47,7 & 0.28 & 12,5 & 3,5 & 16,8 & 488 & 41,3 & 727 & 45,6 & 12,79 \\
\hline $15=6973$ & 43,0 & 0,17 & 9,6 & 2,8 & 12,9 & 500 & 40,7 & 727 & 48,4 & 12,66 \\
\hline $16=6974$ & 43,9 & 0,42 & 13,2 & 3,7 & 17,9 & 476 & 70,7 & 735 & 48,0 & 12,71 \\
\hline $17=6975$ & 43,9 & 0,49 & 12,3 & 3,3 & 16,7 & 489 & 42,2 & 752 & 46,0 & 9,51 \\
\hline $18=6978$ & 38,5 & 0,51 & 13,6 & 4,0 & 18,8 & 494 & 42,2 & 770 & 43,4 & 10,61 \\
\hline $19=6990$ & 44,6 & 0,17 & 11,1 & 2,6 & 14,2 & 448 & 41,1 & 758 & 34,1 & 10,46 \\
\hline $20=6991$ & 41.6 & 0.24 & 10,7 & 2,7 & 14,1 & 488 & 41,1 & 758 & 34,1 & 10,46 \\
\hline $21=7029$ & 20,0 & 0,10 & 7,0 & 3,2 & 10,6 & 584 & 40,3 & 754 & 38,4 & 10,73 \\
\hline $22=7046$ & 22,9 & 0,10 & 7,3 & 2,8 & 10,5 & 523 & 40,4 & 764 & 35,6 & 11,32 \\
\hline $23=7080$ & 17,9 & 0,36 & 6,4 & 2,7 & 9,6 & 590 & 39.8 & 737 & 35,8 & 9,65 \\
\hline $24=7116 \mathrm{~A}$ & 17.3 & 0.15 & 6,9 & 3,0 & 9,5 & 592 & 40,4 & 783 & 37,0 & 9,66 \\
\hline $25=7116 \mathrm{~B}$ & 17,4 & 0,30 & 6,5 & 3,5 & 10,6 & 584 & 40,0 & 788 & 33,3 & 9,67 \\
\hline Moyennes & 35,4 & 0,30 & 9,7 & 3,2 & 13.1 & 516 & 40,9 & 750 & 40,8 & 11,1 \\
\hline$C v_{p}(\%)$ & 32.1 & 47,4 & 22,6 & 14,2 & 22,3 & 8,5 & 2.0 & 3,5 & 10,6 & 10.2 \\
\hline
\end{tabular}

labre pour la longueur des fibres). Ceci nous amène à poser la question de savoir s'il est valable de sélectionner pour ces 2 caractères.

La circonférence varie de 28,1 à $43,7 \mathrm{~cm}$ en Sicile avec un coefficient de variation de $12,2 \%$. Elle ne présente pas de variabilité infraspécifique significative en Calabre ; pourtant, l'intervalle entre les provenances extrêmes va de 33,3 à $48,4 \mathrm{~cm}\left(C V_{p}=10,6 \%\right)$. La hauteur varie de 5,9 à $9,9 \mathrm{~m}$ en $\mathrm{Si}$ cile $\left(C V_{p}=13,4 \%\right)$ et de 9,5 à $13,1 \mathrm{~m}$ $\left(C V_{p}=10,2\right)$ en Calabre. Ces résuitats confirment la variabilité de la vigueur déjà soulignée par de nombreux auteurs (Destremau et al, 1973 ; Lacaze, 1977 ; Bellefontaine et al, 1977 ; Emery et Ledig, 1987). Ces derniers auteurs ont montré qu'en plus de la 
vigueur, la rectitude, la fourchaison et la floraison présentaient aussi une variabilité infraspécifique significative. La floraison est un critère qui a son importance dans les pays comme le Maroc où la culture des Eucalyptus est souvent associée à l'apiculture qui contribue fortement à la rentabilité des plantations.

Cette variabilité existe aussi au niveau des régions de provenances. Si, en particulier, nous comparons les moyennes des 16 provenances originaires de l'Australie méridionale et du bassin de la rivlère Murray aux moyennes des 9 provenances des autres régions (Queensland, Territoire du Nord et Australie occidentale), nous constatons (tableau $\mathrm{V}$ ) que les premières se distinguent nettement des autres par des retraits après reconditionnement importants, une vigueur élevée, une longueur des fibres faible et des rendements papetiers comparables.

L'Australie méridionale et le bassin de la rivière Murray sont les régions gé- néralement prospectées pour les graines. Les provenances originaires de ces régions se caractérisent par une vigueur élevée par rapport à celles des autres régions. Les provenances de ces dernières se sont avérées plus intéressantes au point de vue qualité du bois. Cependant, certaines d'entre elles sont peu adaptées aux conditions méditerranéennes.

Si l'on reprend les chiffres de l'étude portant sur le retrait (Sesbou et Nepveu, 1978 op cit), on notera que le reconditionnement, en permettant la récupération d'une grande partie du collapse, fait diminuer ces valeurs de façon spectaculaire pour arriver à des retraits compatibles avec beaucoup d'usages. C'est ainsi qu'après cette opération, les valeurs du retrait ne varient plus que de 10,0 à $19,1 \%$ en Sicile et de 9,5 à $18,8 \%$ en Calabre. Le reconditionnement est plus efficace pour les provenances à retrait élevé (bois à vigueur élevée). Ainsi la provenance 10 avait-elle, en Sicile, un retrait

Tableau V. Comparaison des moyennes des provenances originaires du bassin de la rivière Murray $\left(X_{1}\right)$ aux moyennes des provenances des autres régions $\left(X_{2}\right)$.

\begin{tabular}{|c|c|c|c|c|c|c|}
\hline Stations & & Sicile & & $\mathrm{Ca}$ & abre & + \\
\hline Caractères & Test $F$ & $x_{1}$ & $x_{2}$ & Test $F$ & $x_{1}$ & $x_{2}$ \\
\hline Retrait volumétrique (\%) & $121,3 \star \star$ & 27,43 & 51,66 & $179,0 \star \star$ & 21,45 & 43,29 \\
\hline $\begin{array}{l}\text { Retrait volumétrique } \\
\text { après reconditionnement (\%) }\end{array}$ & $42,6 * *$ & 11,24 & 15,68 & $28,6 * *$ & 11,02 & 14,99 \\
\hline Infradensité $\left(\mathrm{kg} / \mathrm{m}^{3}\right)$ & $25,0 \star \star$ & 537 & 483 & $54,3 \star \star$ & 564 & 489 \\
\hline Rendement papetier (\%) & $0,1 \mathrm{NS}$ & 41,48 & 41,62 & $2,0 \mathrm{NS}$ & 40,61 & 41,08 \\
\hline Longueur des Fibres $(\mu \mathrm{m})$ & $15,9 \star *$ & 763 & 732 & $8,8 * *$ & 768 & 740 \\
\hline Circonférence $(\mathrm{cm})$ & $41,2^{\star \star}$ & 31,8 & 39,2 & $14,6 * \star$ & 37.29 & 42.77 \\
\hline Hauteur $(m)$ & $28,3 \star \star$ & 7.05 & 8,70 & $1,3 \mathrm{NS}$ & 10,76 & 11,31 \\
\hline
\end{tabular}


volumétrique avec collapse de $60 \%$; après reconditionnement, il est passé à $18 \%$ alors que la provenance 24 qui avait le faible retrait avec collapse (22 \%) a atteint après reconditionnement $12 \%$. Certaines provenances mal classées avant le reconditionnement ont moins bien réagi au traitement que la provenance 10. C'est le cas de la provenance 14 qui est passée de 47,5 à $18,1 \%$. Mais, en genéral, les classements des provenances avant et après reconditionnement sont peu perturbés car l'on a de bonnes corrélations entre les retraits avant et après traitement ( $r=0,820^{\star \star}$ pour la Sicile, $r=0,758^{\star \star}$ pour la Calabre).

\section{Cas particulier du rendement papetier}

Les plantations d'Eucalyptus camaldulensis réalisées à travers le monde ont surtout pour but la production du bois pour la pâte à papier. A titre d'exemple, au Maroc, $55 \%$ du volume produit par cette essence sont destinés à alimenter une usine de pâte à papier. C'est pourquoi nous nous penchons tout particulièrement sur ce point en envisageant différents scénarios possibles.

Dans le domaine papetier, l'industriel achète le bois soit au poids, soit au volume. Dans le premier cas, il va s'intéresser au rendement en fibres par rapport au poids anhydre du bois qu'il traite. Pour un même poids de matière première, plus ce rendement est grand plus la quantité de pâte produite sera élevée. Dans le second cas, ce qui importe pour lui c'est le rendement en pâte par rapport au volume vert du bois. A volume de bois vert (sur pied) égal, plus la quantité de pâte est grande plus le poids de pâte produite sera élevé. Le rendement en pâte par rapport au volume de bois vert $\left(R_{b v}\right)$ est obtenu par:

$R_{b v}=$ Densité basale $\times$ rendement papetier
Le propriétaire forestier dont le principal objectif est de tirer le meilleur revenu de ses peuplements s'interesse a la production en pâte à l'hectare. Cette dernière peut être évaluée, soit par le rendement en pâte à l'hectare $\left(R_{h a}\right)$, soit par le rendement en pâte à l'arbre moyen $\left(R_{a m}\right)$. On aura donc :

- Rendement en pâte à l'hectare :

$R_{h a}=$ rendement papetier $\times$ densite basale $\times V_{\text {ha }}$

$V_{\text {ha }}$ étant la production à l'hectare.

- Rendement en pâte à l'arbre moyen : $R_{a m}=$ rendement papetier $\times$ densité basale $\times V_{a m}$

$V_{a m}$ étant le volume de l'arbre moyen. Nous avons comparé le classement des provenances en envisageant les 3 critères suivants : rendement par rapport au poids anhydre, rendement par rapport au volume vert, rendement par rapport à l'arbre moyen. Pour ce dernier cas, le volume de l'arbre moyen par provenance est calculé à partir de la circonférence (C) et de la hauteur $(\mathrm{H})$ moyennes de la provenance :

$V_{a m}=\left(C^{2} / 4 \Pi\right) \times H \times K$

Ne disposant pas des valeurs du coefficient de forme $(K)$ pour chacune des provenances, nous avons pris un coefficient égal à 1 pour toutes les provenances. $R_{a m}$ n'a donc qu'une valeur relative car il permet de situer les provenances les unes par rapport aux autres. Les figures 1 et 2 donnent les classements des provenances suivant ces 3 critères respectivement en Sicile et en Calabre.

Si on ne tient compte que du rendement par rapport au poids de bois anhydre, 4 provenances se distinguent par leurs performances dans les 2 stations : 2 (Shepparton), 8 (Petford), 17 et 18 (descendances Port Lincoln). En Calabre, la provenance 20 (Walpola island State Forest) s'ajoute aux 4 premières. Le classement en fonction du 

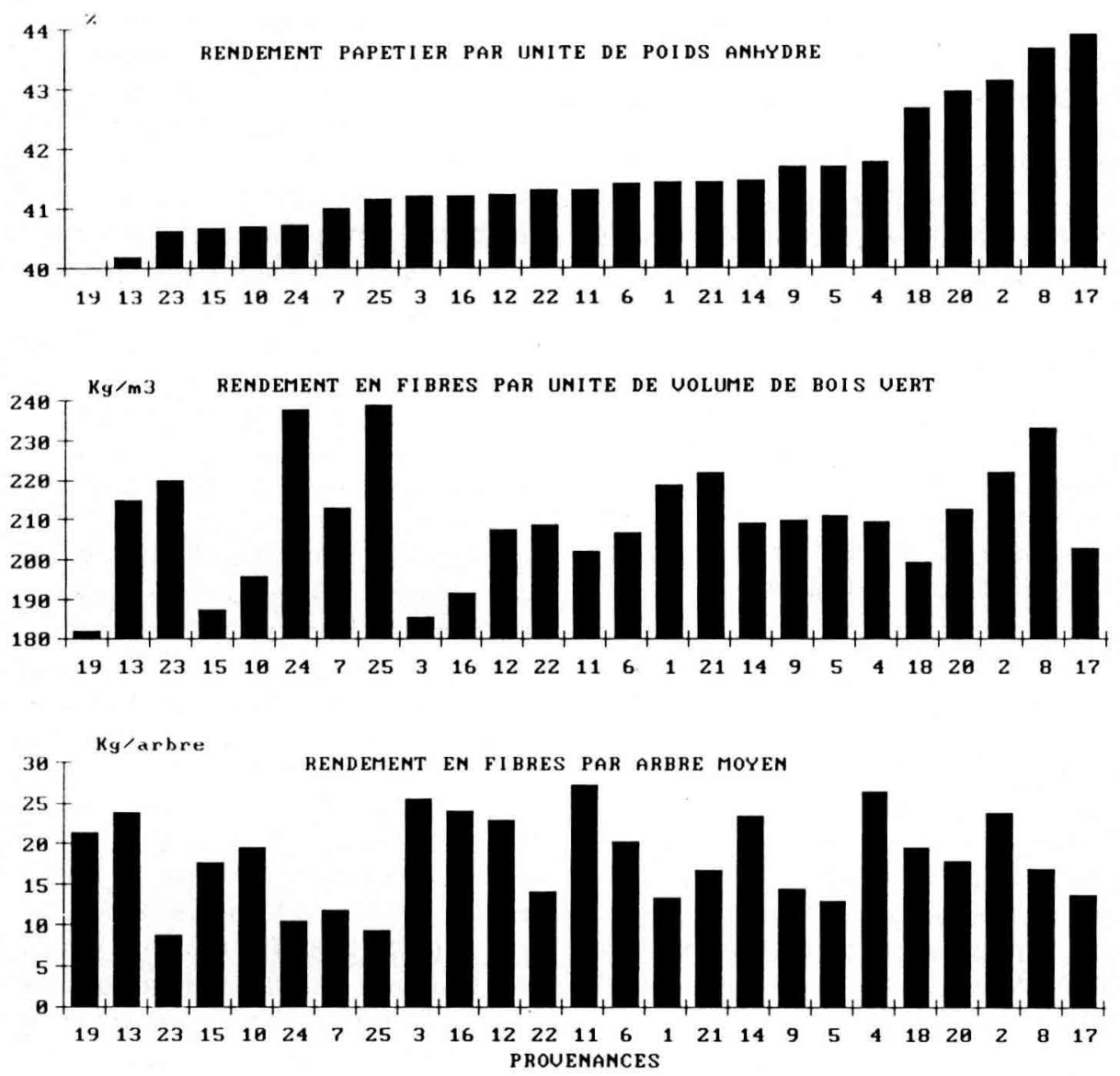

Fig 1 Rendement en fibres par rapport au poids du bois anhydre, au volume de bois vert et par arbre moyen pour les différentes provenances en Sicile.

rendement par rapport au volume de bois vert donne un résultat différent. On voit apparaître les provenances à densité élevée : 21 (Murchison River), 23 (New Castle Waters Creek), 24 et 25 (Tennant Creek). Seule la provenance 8 (Petford) se maintient en bonne position. Le rendement par rapport à l'arbre moyen permet un classement complètement différent des 2 premiers. Les provenances qui arrivent en tête sont : 4 (Lake Albacutya), 14, 15 et 16 (descendances de la prove- nance Est Adélaide) et 8 (Petford) en Sicile et 3 (Woohlpooer State Forest), 4 (Lake Albacutya), 11, 14 et 16 (descendances de la provenance Est Adélaide) en Calabre.

L'améliorateur devrait prendre en compte tous ces caracteres pour que la sélection atteigne pleinement son but. Nous voyons notamment que si l'on omettait de prendre en considération la vigueur, l'on négligerait des provenances intéressantes. Deux autres caractéristiques auraient été également 

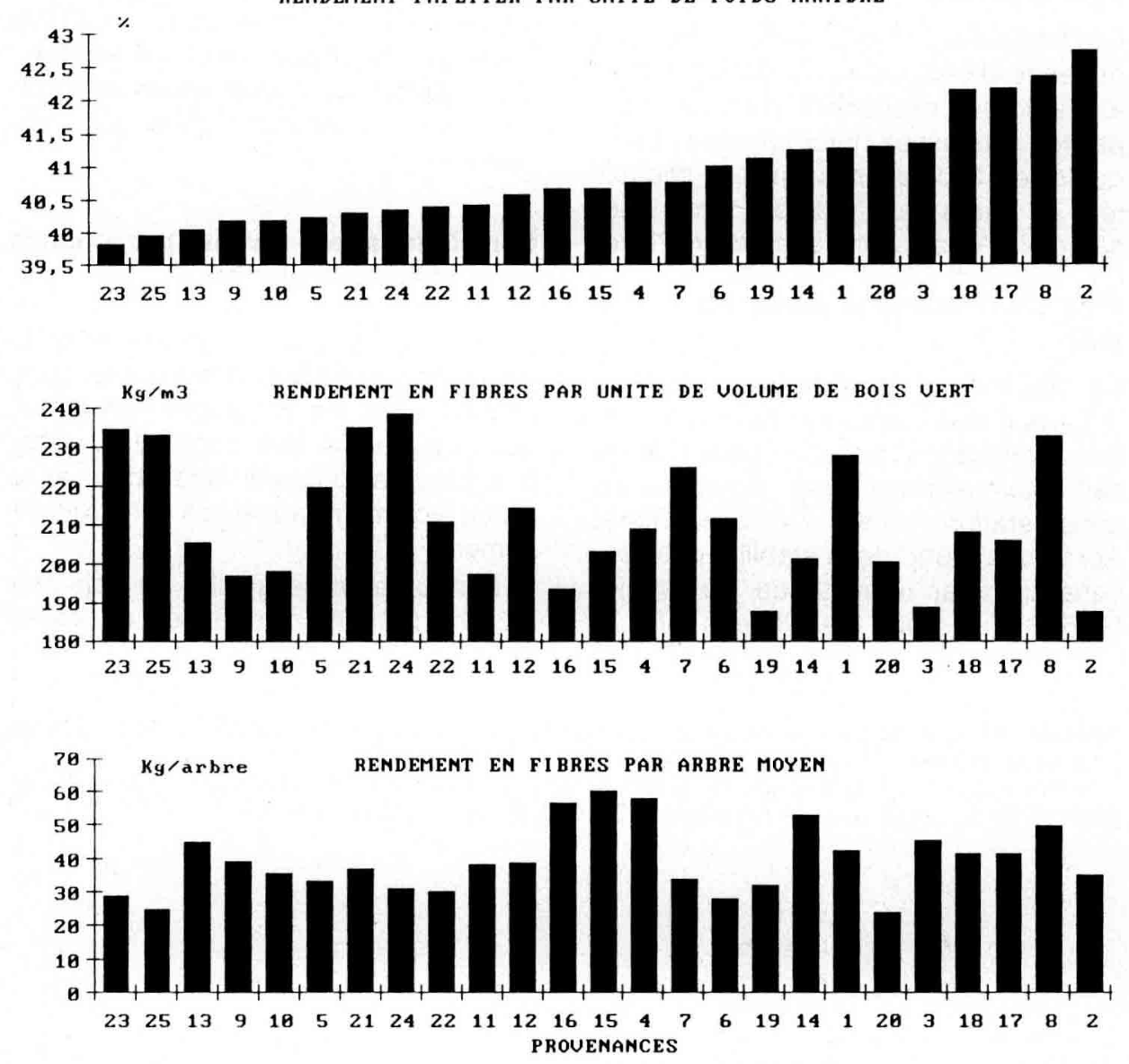

Fig 2 Rendement en fibres par rapport au poids du bois anhydre, au volume de bois vert et par arbre moyen pour les différentes provenances en Calabre.

à considérer pour envisager tous les aspects de la sélection pour la production papetière : le taux d'écorce et l'importance des extraits. Nous n'en disposions malheureusement pas.

Les résultats obtenus illustrent l'intérêt d'une sélection infraspécifique. Nous n'avons rien trouvé dans la littérature sur la variabilité infraspécifique de la qualité du bois de l'Eucalyptus camaldulensis. Cependant, nous trou- vons des données sur la variabilité génétique individuelle pour d'autres espèces d'Eucalyptus. Ainsi, l'héritabilité génotypique de la densité du bois a-telle été calculée pour d'autres espèces du genre Eucalyptus. Elle est très forte pour $E$ grandis en Australie (Bamber et Humphreys, 1963), pour $E$ regnans (Higgs, 1969), pour $E$ deglupta (Davidson, 1972) et pour E globulus (Celbi, 1979). 


\section{Effet répétition}

Les résultats obtenus (effet positif ou absence d'effet selon les caractères et la station) s'expliquent probablement par les sols assez hétérogènes sur lesquels les 2 dispositifs ont été implantés : pente en Calabre, manteau sableux d'épaisseur variable en Sicile.

Effet de l'interaction provenance-répétition :

La présence d'un effet significatif sur la plupart des caractères pour l'interaction provenance-répétition nous interdisait normalement de prendre en consideration l'effet "provenance". Après une étude de la stabilité des provenances par la méthode de Wricke (1962) (que nous détaillerons plus à fond au sujet des interactions provenance $\times$ station), nous constatons que l'effet de l'interaction est dû à l'instabilité de quelques provenances en fonction des répétitions (Sesbou, non publié).

\section{Corrélations au niveau provenance dans chaque dispositif}

Dans le tableau VI, nous avons fait figurer les corrélations entre les facteurs étudiés dans les 2 stations. En Sicile, nous observons une corrélation négative entre la longueur des fibres et le retrait volumétrique après reconditionnement.

Les facteurs de qualité du bois étudiés ne présentent que peu de corré-

Tableau VI. Coefficients de corrélations entre moyennes de provenances pour la qualité du bois et la vigueur en Sicile et en Calabre.

- Signifie que la circontérence ne présente pas de variabilité infraspécifique. Seuils de signification du coefficient de corrélation (23 degrés de liberté) $0,396(5)=* ; 0,505(1)=$ **

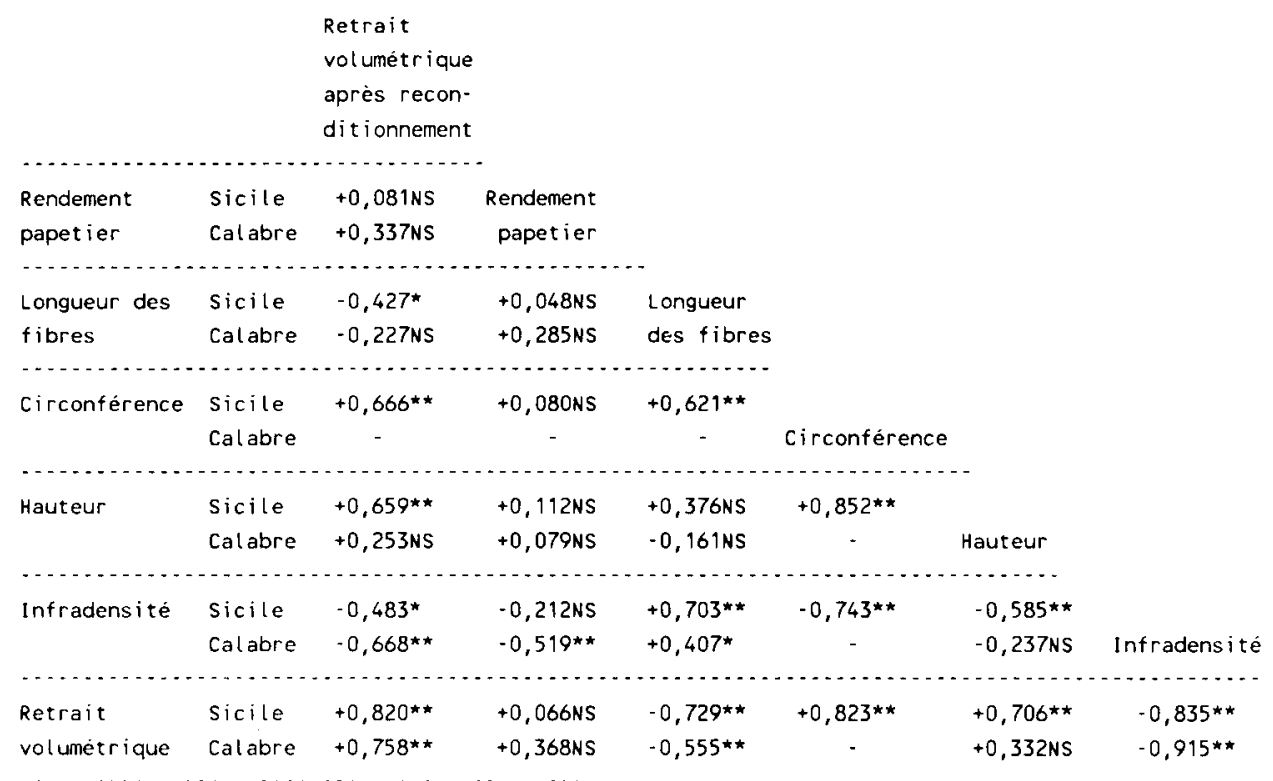


lations avec les critères de vigueur. Nous notons toutefois que le retrait volumétrique après reconditionnement présente des corrélations positives avec la circonférence et la hauteur en Sicile.

Si l'on tient compte dans l'étude des corrélations des paramètres étudiés dans un précédent article (Sesbou et Nepveu, 1978, op cit) à savoir l'infradensité et le retrait volumétrique avec collapse, les résultats suivants apparaissent : le retrait volumétrique avant reconditionnement présente une liaison positive très significative avec le retrait volumétrique après reconditionnement et une liaison négative avec la longueur des fibres dans les 2 stations. L'infradensité présente des corrélations négatives avec les retraits volumétriques avant et après reconditionnement et positives avec la longueur des fibres dans les 2 stations. Elle est corrélée négativement avec le rendement papetier en Calabre seulement.

Au niveau provenance, une bonne vigueur s'accompagne, en Sicile, de retraits avant et après reconditionnement importants et d'une infradensité faible.

Nous avons montré qu'il existait des liaisons entre certains caractères au niveau des provenances. Ces liaisons font que toute augmentation de la vigueur s'accompagne en Sicile d'une augmentation des retraits et d'une diminution de l'infradensité.

Ceci éclaire d'un jour nouveau la stratégie d'amélioration de Eucalyptus camaldulensis, basée jusqu'ici sur la vigueur, si l'on considère que le retrait volumétrique sans reconditionnement peut varier de 22 à $60 \%$ barrant ainsi la route à l'utilisation noble de certaines origines.

Jusqu'à présent, cette stratégie visait la production du bois de trituration. En cas de changement vers la produc- tion de bois d'œurre, le sylviculteur aura à choisir, soit les provenances à croissance lente, soit les provenances à croissance rapide avec des pratiques sylvicoles appropriées.

\section{Étude de l'effet station}

\section{Comparaison de moyennes entre les 2 stations :}

Nous avons comparé les moyennes de provenances en Sicile et en Calabre par la méthode des couples (tableau VII). L'effet station est significatif pour tous les caracteres a l'exception des retraits longitudinal et volumétrique après reconditionnement et de la longueur des fibres.

En Sicile, les valeurs du retrait radial après reconditionnement et de l'infradensité sont inférieures à celles de la Calabre ; les valeurs des autres caractères sont supérieures. A ce niveau, ces résultats démontrent une liaison négative retraits-infradensité et une liaison positive rendement-retrait tangentiel. Il en va autrement pour la corrélation infradensité-vigueur. En passant de la Sicile à la Calabre, les retraits et le rendement papetier diminuent alors que l'infradensité et la vigueur augmentent.

Notons cependant, qu'en passant d'une station pauvre à une autre plus fertile, nous avons, tout en augmentant la circonférence de $8,1 \%$, la hauteur de $37,8 \%$, le retrait radial après reconditionnement de $7,5 \%$ et l'infradensité de $2,6 \%$, diminué le retrait tangentiel après reconditionnement de $8,1 \%$ et le rendement papetier de $1,5 \%$. 
Tableau VII. Effets de la station sur les différents caractères (comparaison des moyennes par la méthode des couples). Valeur du $t$ calculé à 24 degrés de liberté: 2,10 (5\%); 2,80 $(1 \%)$.

\begin{tabular}{lccc}
\hline \multicolumn{1}{c}{ Caracteres } & $\begin{array}{c}\text { Valeur dut } \\
\text { calculé (24 ddl) }\end{array}$ & $\begin{array}{c}\text { Moyenne } \\
\text { Sicile }\end{array}$ & $\begin{array}{c}\text { Moyenne } \\
\text { Calabre }\end{array}$ \\
\hline & & & \\
Retrait longitudinal après reconditionnement & $1,26 \mathrm{NS}$ & $0,33 \%$ & $0,29 \%$ \\
Retrait tangentiel après reconditionnement & $3,68^{\star \star}$ & $10,55 \%$ & $9,70 \%$ \\
Retrait radiat après reconditionnement & $2,69 *$ & $2,94 \%$ & $3,16 \%$ \\
Retrait volunetrique après reconditionnernent & $1,97 \mathrm{NS}$ & $14,22 \%$ & $13,53 \%$ \\
Rendenent papetier & $4,26 \star \star$ & $41,56 \%$ & $40,93 \%$ \\
Longueur des fibres & $1,45 \mathrm{NS}$ & $0,742 \mathrm{~mm}$ & $0,748 \mathrm{~mm}$ \\
Circonférence & $5,73^{\star \star}$ & $36,6 \mathrm{~cm}$ & $40,5 \mathrm{~cm}$ \\
Hauteur & $14,86^{\star \star}$ & $8,17 \mathrm{~cm}$ & $11,26 \mathrm{~cm}$ \\
Infradensité & $3,45^{\star \star}$ & $503 \mathrm{~kg} / \mathrm{mm}^{3}$ & $516 \mathrm{~kg} / \mathrm{m}^{3}$ \\
\hline
\end{tabular}

\section{Interaction génotype-environnement :}

Pour exploiter une forte variabilité infraspécifique de façon économique (c'est-à-dire sans compliquer le programme d'amélioration), il faut que les interactions provenance $x$ station soient faibles. Pour tester la stabilité des provenances dans les stations étudiées, nous avons utilisé le paramètre de stabilité de Wricke (1962) : l'écovalence dont Kremer (1976) a donné une application dans le cas du pin maritime.

Une provenance possede une haute écovalence (une bonne stabilité) si, dans chaque test, son classement par rapport à la moyenne générale de toutes les provenances est relativement stable. Quantitativement, ceci equivaut à mesurer la contribution de cette provenance à la somme des carrés des ecarts du terme de l'interaction provenance $x$ station dans l'analyse de variance. Cette contribution se calcule par l'interactivité de la provenance $i$ $\left(W_{i}\right)$ qui est l'inverse de l'écovalence :

avec :

$$
W_{i}=\sum_{j} n_{i j}\left(x_{i j}-x_{i .}-x_{. j}-x_{. .}\right)^{2}
$$

$X_{i j}=$ moyenne de la provenance $i$ dans le milieu $j$

$X_{i}=$ moyenne de la provenance $i$ dans tous les milieux

$X_{. j}=$ moyenne de toutes les provenances dans le milieu $j$

$X$. = moyenne générale

$n_{i j}=$ nombre d'individus de la provenance $i$ dans le milieu $j$

La somme de tous les $W_{i}$ est égale à la somme des carrés de l'interaction provenance $\times$ station. On peut donc exprimer chaque $W_{i}$ sous forme d'une contribution en $\%$ à l'interaction provenance $\times$ station. Ceci permet de détecter les provenances stables et celles qui le sont moins.

Nous nous limiterons aux principaux caractères à savoir le retrait volumétrique, le retrait volumétrique après re- 
conditionnement, l'infradensité du bois et le rendement papetier. Le tableau VIII donne le classement et l'écovalence des différentes provenances pour les caractères étudiés.

\section{Le retrait volumétrique}

Cinq provenances $(10,12,13,14$ et 18) se distinguent par leur instabilité d'une station à une autre. Elles contribuent pour $56,4 \%$ à la somme des carrés des écarts de l'interaction alors que 7 autres provenances $(2,4,6,9,21,23$ et 24) participent ensemble pour moins de $2 \%$. Les provenances 13 et 14 ont un retrait plus faible par rapport à la moyenne expérimentale quand on passe de Calabre en Sicile. Elles étaient classées respectivement $15^{\mathrm{e}}$ et $23^{e}$ en Calabre. Leurs classements s'améliorent en Sicile : $10^{\ominus}$ et $14^{\ominus}$. II en est autrement des provenances 10,12 et 18. Leurs rangs de classement sont meilleurs en Calabre qu'en Sicile grâce

Tableau VIII. Classement et écovalence des provenances pour les retraits volumétriques, l'infradensité et le rendement papetier.

\begin{tabular}{|c|c|c|c|c|}
\hline $\begin{array}{c}\text { Prove- } \\
\text { nances }\end{array}$ & $\begin{array}{l}\text { Retrait volumetrique } \\
\text { avec collapse }\end{array}$ & $\begin{array}{c}\text { Retrait volumetrique } \\
\text { apres recondition- } \\
\text { nement }\end{array}$ & Infradensité & $\begin{array}{c}\text { Rendement } \\
\text { papetier }\end{array}$ \\
\hline
\end{tabular}

Sicile Calabre \% $\Sigma W_{i j}$ Sicile Calabre \% $\Sigma W_{i j}$ Sicile Calabre\% $\Sigma W_{i j}$ Sicile Calabre\% $\Sigma W_{i j}$

\begin{tabular}{|c|c|c|c|c|c|c|c|c|c|c|c|c|}
\hline 1 & 19 & 9 & 1,3 & 9 & 4 & 2,9 & 7 & 5 & 3,41 & 10 & 7 & 1,5 \\
\hline 2 & 124 & 25 & 0,0 & 16 & 14 & 0,7 & 24 & 25 & 7.11 & 3 & 1 & 0,4 \\
\hline 3 & 16 & 24 & 1.7 & 14 & 17 & 1,7 & 25 & 23 & 1,41 & 17 & 6 & 4,2 \\
\hline 4 & 15 & 17 & 0,3 & 18 & 12 & 4,6 & 13 & 13 & 0,21 & 6 & 11 & 1,0 \\
\hline 5 & 18 & 8 & 1,8 & 8 & 13 & 9,4 & 11 & 7 & 6,61 & 7 & 21 & 5,8 \\
\hline 6 & 12 & 10 & 0,6 & 11 & 7 & 1,3 & 14 & 11 & 0,61 & 12 & 10 & 0,3 \\
\hline 7 & 15 & 6 & 1,2 & 2 & 8 & 4,2 & 8 & 8 & 1,2 & 19 & 11 & 1,1 \\
\hline 8 & 3 & 5 & $4,8 \mid$ & 7 & 9 & 1,2 & 4 & 6 & 0,01 & 2 & 2 & 3,3 \\
\hline 9 & 13 & 11 & 0.11 & 15 & 10 & $4,2 \mid$ & 9 & 16 & 9,51 & 8 & 20 & 5,0 \\
\hline 10 & 25 & 22 & 9.71 & 23 & 20 & 4,9 & 18 & 17 & 0.0 & 21 & 22 & 0,0 \\
\hline 11 & 23 & 20 & 4,01 & 22 & 18 & 6,6 & 16 & 15 & 0,0 & 13 & 16 & 0,4 \\
\hline 12 & 19 & 13 & 11,6 & 25 & 19 & 18,2 & 14 & 10 & 3,0 & 15 & 15 & 0,0 \\
\hline 13 & 10 & 15 & 13,01 & 17 & 21 & 6.4 & 4 & 11 & $12,3 \mid$ & 24 & 23 & 2,0 \\
\hline 14 & 14 & 23 & $13,3 \mid$ & 24 & 23 & 1,0 & 10 & 19 & 9.51 & 9 & 8 & 1,3 \\
\hline 15 & $\mid 18$ & 16 & $1,6 \mid$ & 12 & 11 & 0,0 & 22 & 18 & 3.81 & 22 & 13 & 2,9 \\
\hline 16 & $\mid 21$ & 18 & $4,1 \mid$ & 20 & 24 & $4,1 \mid$ & 21 & 22 & 0,6 & 16 & 14 & 0,1 \\
\hline 17 & 20 & 19 & 3,3 & 19 & 22 & 3,9 & 20 & 20 & $1,0 \quad \mid$ & 1 & 3 & 8,4 \\
\hline 18 & 17 & 12 & $8,7 \mid$ & 21 & 25 & 9.1 & 19 & 14 & 7,21 & 5 & 4 & 0,1 \\
\hline 19 & 22 & 20 & 3.71 & 13 & 16 & 1.6 & 23 & 24 & 6.61 & 25 & 5 & 47,2 \\
\hline 20 & | 11 & 14 & 4,51 & 10 & 15 & 4,5 & 16 & 21 & 6,01 & 4 & 9 & 9,9 \\
\hline 21 & 6 & 4 & $0,0 \mid$ & 4 & 5 & 0,0 & 6 & 4 & 7.21 & 10 & 19 & 1,6 \\
\hline 22 & 7 & 7 & 2,2 & 1 & 3 & 2,2 & 11 & 9 & $0,8 \mid$ & 14 & 17 & 0,5 \\
\hline 23 & 4 & 3 & 0,41 & 3 & 2 & 0,91 & 3 & 1 & 10,91 & 23 & 25 & 0,1 \\
\hline 24 & 1 & 1 & $1,8 \mid$ & 6 & 1 & 6,2 & 1 & 1 & 1,0 & 20 & 18 & 0,5 \\
\hline 25 & 2 & 2 & 0,31 & 5 & 6 & 0,8 & 2 & 3 & 0,01 & 18 & 24 & 2,0 \\
\hline
\end{tabular}


à une réaction favorable au milieu. La provenance 2 qui présente la plus forte écovalence est très mal classée dans les 2 stations. Les provenances 23,24 et 25 présentent à la fois des retraits faibles et une écovalence élevée.

\section{Le retrait volumétrique après recondi- tionnement}

Six provenances $(5,11,12,13,18$ et 24) représentent $56 \%$ de la somme des carrés des écarts de l'interaction. Nous retrouvons les provenances 12 , 13,18 et 24 qui présentaient des écovalences faibles pour le retrait volumétrique. Les provenances 11,12 et 24 accusent une amélioration du retrait volumétrique quand nous passons de Sicile en Calabre. Pour les 3 autres provenances, nous assistons au phénomène inverse : les retraits en Calabre sont supérieurs aux retraits en Sicile. II en résulte une aggravation de leurs classements respectifs. Quand nous comparons la stabilité de la provenance 18 avant et après reconditionnement, nous constatons que dans le premier cas, nous avions une amélioration du retrait volumétrique quand nous passions de Sicile en Calabre et dans le second, nous avions l'inverse, à savoir une aggravation du retrait. Notons au passage qu'il s'agit d'une provenance qui ne réagit pas au reconditionnement.

Les provenances les plus stables sont les $2,6,15,21$ et 25 .

\section{L'infradensité}

Nous avons, pour ce critere, des provenances qui présentent une stabilité remarquable. C'est le cas notamment des provenances $4,6,8,10,11,16$, $17,22,24$ et 25 dont la participation à l'interaction ne dépasse pas 4,5\% de la variance totale. D'un autre côté, nous avons 7 provenances $(2,9,13$,
14, 18, 21 et 23) qui, à elles seules contribuent pour $64 \%$ environ à l'interaction. Quand nous passons de Sicile en Calabre, nous avons, par rapport à la moyenne expérimentale, une augmentation de la densité chez les provenances $2,9,13$ et 14 .

La provenance 3 qui participe pour $1,4 \%$ à l'interaction est très mal classée dans les 2 stations.

\section{Le rendement papetier}

Pour ce caractère, le problème est plus simple car une seule provenance, la 19 , contribue pour $47 \%$ a l'interaction. De dernière en Sicile, elle devient $5^{\mathrm{e}}$ en Calabre. Les provenances 17 et 20 passent respectivement de 1 et 4 en Sicile, à 3 et 9 en Calabre et contribuent ensemble pour $18 \%$ à la somme des carrés des écarts de l'interaction; soit $65,5 \%$ pour les 3 provenances.

Les provenances les plus stables sont, dans l'ordre, les provenances 10 , $12,16,18,23,6$ et 2 . Cette dernière est la plus performante alors que la 23 est la plus mal classée des provenances les plus stables.

\section{Stabilité des provenances pour tous les caractères}

$\mathrm{Si}$ nous considérons la stabilité des provenances au niveau de tous les caractères, nous nous apercevons que certaines provenances présentent une bonne stabilité dans les 2 stations. II s'agit des provenances $3,7,16,24$ et 25 . Les plus intéressantes pour la qualité du bois étant les provenances 24 et 25 qui présentent un retrait faible, une infradensité et une longueur des fibres élevées. Malheureusement, leur vigueur ainsi que leur rendement papetier sont médiocres. Les autres provenances sont plus ou moins stables suivant les caractères, la 19 etant la plus instable. 
Pour bien tester la stabilité des différentes provenances, il faut, comme l'a déjà signalé Lacaze (1977), installer des dispositifs dans des conditions marginales (étage aride, variante froide du subhumide), ce qui permettrait de déceler l'aptitude de certaines provenances a supporter des conditions particulières.

Enfin, les plantations d'Eucalyptus camaldulensis étant le plus souvent traitées en taillis, il serait intéressant de compléter ce travail par une étude sur la variabilité de la qualité du bois dans les différentes rotations du taillis pour suivre l'évolution de la qualité du bois, notamment le classement relatif des provenances à ces différents stades.

\section{CONCLUSION}

L'Eucalyptus camaldulensis se caractérise par une importante variabilité infraspécifique tant au niveau de la qualité du bois que de la vigueur. Ceci confirme bien les résultats d'un précédent article portant sur le même matériel végétal mais dans lequel nous avions pris des propriétés du bois différentes. Son importance varie en fonction des caractères étudiés : très élevée pour les retraits et la vigueur et faible pour le rendement papetier et la longueur des fibres.

Cette variabilité permet d'ores et déjà d'envisager une sélection très efficace au niveau infraspécifique. Cependant, l'efficacité de cette sélection serait meilleure si, au lieu de sélectionner sur un seul caractère, nous sélectionnions sur un indice qui intégrerait tous les caracteres du bois et de la vigueur qui contribuent à la qualité du bois pour une utilisation donnée. Nous avons montré que, pour l'Eucalyptus camaldulensis à usage papetier, la sélection doit porter sur un indice qui tient compte de la densité, du rendement papetier et de la vigueur.

Cette étude a permis de mettre en évidence d'autres données intéressantes à savoir :

- Le rôle que joue le reconditionnement dans la récupération du collapse et son efficacité pour les provenances à fort retrait (vigueur élevée).

- L'intérêt pour le reboiseur à utiliser les meilleurs sites pour augmenter la vigueur tout en améliorant la qualité du bois, en particulier diminuer le retrait du bois.

- L'existence de provenances stables quand elles sont plantées dans des milieux variés. Notons toutefois que certaines provenances présentent une bonne stabilité pour certains caractères seulement.

- L'existence d'une région de provenances homogène, le bassin de la rivière Murray et l'Australie méridionale, région qui se caractérise par rapport aux autres régions par des densités et des longueurs de fibres faibles, des retraits et une vigueur élevés. Pour le rendement papetier, on n'a pas de différence entre groupes de régions.

\section{RÉFÉRENCES}

Bamber RK, Humphreys FR (1963) A preliminary study of some wood properties of Eucalyptus grandis (Hill) Maiden. J Inst Wood Sci, 11, 63-70

Bellefontaine R, Raggabi $M$, Nanson A (1977) Expérience internationale d'origines d'Eucalyptus camaldulensis Dehn. Dispositif de Sidi Slimane (Maroc). Ann Rech For Maroc, Tome 19, 339-389

Celbi (1979) Heridity studies on Eucalyptus globulus. Document FO : FGB 79-3/5. Consultation technique sur les feuillus a croissance rapide pour la plantation dans les zones méditerranéennes et tempérées. Lisbonne 
Davidson J (1972) Variation, association and inheritance of morphological and wood characters in an improvment program for Eucalyptus deglupta Blum Ph D Thesis, Aust Nat Univ $262 \mathrm{pp}$

Destremau DX, Jolly H, Korba M (1973) Les enseignements de l'essai comparatif de provenances d'Eucalyptus camaldulensis de Sidi Slimane. Ann Rech For Maroc, 13, 121-154

Emery BM, Ledig FT(1987) Provenance variation in Eucalyptus calmadulensis Dehn in California Sivlae Genet 36, 172-180

Ferrand JC (1981) Recherches sur les solutions pratiques à apporter aux problèmes posés par les contraintes de croissance des arbres forestiers. Thèse de docteuringénieur en sciences du bois, Institut national polytechnique de Lorraine, Nancy, $206 \mathrm{pp}$

Higgs ML (1969) Genetic and environmental factors influencing commercially important wood properties of Eucalyptus grandis FvM Ph D Thesis, Aust Nat Univ

Janin G (1972) Microcuissons papetières. Méthode adaptée aux recherches forestières portant sur la détermination des caractéristiques papetières individuelles sur arbres vivants à l'aide d'échantillons de bois dont le mode de prélèvement, l'aspect et le poids ne sont pas usuels. Papeterie, $\mathrm{n}^{\circ} 3,174-188$

Janin G, Rahme A (1972) Étude de la variabilité des rendements en fibres sur Pinus brutia. Document à usage interne $n^{\circ} 1972 / 3$. Stat rech qual bois. CNRF. Champenoux.

Keller R (1973) Caractéristiques du bois de pin maritime. Variabilité et transmission héréditaire. Ann Sci For, 30, 63-81

Keller R et Thoby M (1977) Liaisons entre l'état juvénile et l'état adulte pour quelques caractères technologiques et auxométriques chez le Douglas (Pseudotsuga menziesii). Ann Sci For, 34, 175203

Kremer A (1976) Contrôle génétique de la croissance en hauteur chez le pin maritime : rôle des interactions entre gènes et de l'interaction génotype " $x$ " environnement. Mémoire de fin d'étude de l'école nationale des ingénieurs des Travaux des Eaux et Forêts, Nogent-surVernisson, 107pp
Lacaze JF (1970) Compte rendu operationnel. Projet $n^{\circ} 6$ : Etude de l'adaptation écologique des Eucalyptus. FO : SCM/FR 70/2/10. 24pp

Lacaze JF (1977) Étude de l'adaptation écologique des Eucalyptus. Étude des provenances d'Eucalyptus camaldulensis. Projet FAO $n^{\circ} 6.3^{e}$ consultation mondiale sur l'amélioration des arbres forestiers. Canberra, 25pp

Lacaze JF (1978) Progrès dans le domaine de la sélection d'espèces et de provenances. Unasylva, 30 (119-120), 17-20

Nanson $A$, de Jamblinne $A$, Istas $R_{\text {, Schalck }}$ $J(1975 a)$ Hérédité de la densité du bois chez le pin de Koekelare. Bull Soc R For Belg 82, 68-79

Nanson A, Sacre E, Fraipont L (1975b) Tests précoces de la qualité du bois de provenances d'épicéa commun. Bull Rech Agron Gembloux. 10, 527-558

Nepveu G (1973) Étude génétique de quelques qualites du bois de pin maritime. Corrélations avec des caractères de croissance et d'aptitude à l'élagage. Document à distribution limitée $n^{\circ}$ 1973/5. Stat rech qual bois. CNRF, Champenoux.

Nepveu G (1986) Faut-il se préoccuper des propriétés du bois dans le cadre des programmes d'amélioration génétique des arbres forestiers? Rev For Fr, 28, N spéc, 221-227

Polge H (1973) Facteurs écologiques et qualité du bois. Ann Sci For, 30, 307-332

Polge H, Illy G (1967) Observations sur l'anisotropie du pin maritime des Landes. Ann Sci For, 24, 205-231

Rudman P (1970) The influence of genotype and environment on wood properties of juvenile Eucalyptus camaldulensis Dehn Silvae Genet, 19, 49-53

Sesbou A (1977) Variabilité infraspécifique du retrait et de l'infradensité du bois chez Eucalyptus camaldulensis. Mémoire du Diplôme d'études approfondies. Université de Nancy I. $69 \mathrm{pp}$

Sesbou A, Nepveu G (1978) Variabilité infraspecifique du retrait avec collapse et de la densité du bois chez Eucalyptus camaldulensis. Ann Sci For, 35, 237-263

Wricke G (1962) Uber eine Erfassung der Ökologischen Strenbreite in Feldversuchen. Z Planzenzücht, 47, 92-96 